Supporting Information

\title{
Catalytic Use of Selenium Electrophiles in Cyclizations
}

Danielle M. Browne, Osamu Niyomura, Thomas Wirth*

School of Chemistry, Cardiff University, Cardiff CF10 3AT, UK

wirth@.cf.ac.uk

\section{General procedure for the synthesis of 4-arylbut-3-enoic acids 1a-1f:}

In a $50 \mathrm{~mL}$ flask $(E)$-tributylstannyl 4-tributylstannyl-butyl-but-3-enoate (1.5 g, $2.26 \mathrm{mmol})$, aryl bromide (0.8 eq.) and tetrakis(triphenylphosphine)palladium ( $3 \mathrm{~mol} \%, 78 \mathrm{mg}, 0.068$ $\mathrm{mmol})$ are dissolved in toluene $(20 \mathrm{~mL})$. The mixture was degassed under vacuum and stirred for $10 \mathrm{~h}$ at $100{ }^{\circ} \mathrm{C}$. After cooling, the stannyl ester was hydrolysed with aq. $1 \mathrm{~N} \mathrm{HCl}$ solution. After extraction with diethyl ether $(3 \times 20 \mathrm{~mL})$, the combined organic layers were treated with aq. $1 \mathrm{~N} \mathrm{NaOH}(20 \mathrm{~mL})$. The aqueous phase was washed with diethyl ether and reacidified with aq. $1 \mathrm{~N} \mathrm{HCl}(20 \mathrm{~mL})$ and extracted with diethyl ether $(3 \times 20 \mathrm{~mL})$. The combined organic phases were dried with $\mathrm{MgSO}_{4}$ and, after removal of the solvent under reduced pressure, the compound was purified by flash chromatography eluting with petroleum ether:ethyl acetate:acetic acid $(90: 8: 2){ }^{1}$

(E)-4-Phenylbut-3-enoic acid (1a)<smiles>O=C(O)CC=Cc1ccccc1</smiles>

${ }^{1} \mathrm{H}$ NMR $\left(250 \mathrm{MHz}, \mathrm{CDCl}_{3}\right): \delta=7.12-7.38(5 \mathrm{H}, \mathrm{m}, \mathrm{H}-6,7,8), 6.45(1 \mathrm{H}, \mathrm{d}, J 15.9 \mathrm{~Hz}, \mathrm{H}-4)$, $6.20(1 \mathrm{H}, \mathrm{dt}, J 15.9,7.0 \mathrm{~Hz}, \mathrm{H}-3), 3.20(2 \mathrm{H}, \mathrm{d}, J 7.0 \mathrm{~Hz}, \mathrm{H}-2) \mathrm{ppm}$.

${ }^{13} \mathrm{C} \mathrm{NMR}\left(31 \mathrm{MHz}, \mathrm{CDCl}_{3}\right): \delta=177.8(\mathrm{C}-1), 136.7$ (C-5), $134.0(\mathrm{C}-4), 128.6(2 \mathrm{C}, \mathrm{C}-7)$, 127.7 (C-8), 126.4 (2C, C-6), 120.8 (C-3), 38.0 (C-2) ppm.

(E)-5-Phenylpent-3-enoic acid (1b) ${ }^{1}$<smiles>O=C(O)C/C=C/Cc1ccccc1</smiles>

${ }^{1} \mathrm{H} \mathrm{NMR}\left(250 \mathrm{MHz}, \mathrm{CDCl}_{3}\right): \delta=7.15-7.35(5 \mathrm{H}, \mathrm{m}, \mathrm{H}-7,8,9), 5.75(1 \mathrm{H}, \mathrm{q}, J 6.9 \mathrm{~Hz}, \mathrm{H}-4)$, $5.62(1 \mathrm{H}, \mathrm{q}, J 7.0 \mathrm{~Hz}, \mathrm{H}-3), 3.40(1 \mathrm{H}, \mathrm{d}, J 6.09 \mathrm{~Hz}, \mathrm{H}-5 \mathrm{a}), 6.30(1 \mathrm{H}, \mathrm{d}, J 6.30 \mathrm{~Hz}, \mathrm{H}-5 \mathrm{~b}) \mathrm{ppm}$. ${ }^{13} \mathrm{C}$ NMR $\left(63 \mathrm{MHz}, \mathrm{CDCl}_{3}\right): \delta=178.8(\mathrm{C}-1), 151.4(\mathrm{C}-6), 140.0(\mathrm{C}-3), 128.6(2 \mathrm{C}, \mathrm{C}-7)$, 128.4 (2C, C-8), 126.3 (C-4), 122.5 (C-9), 40.0 (C-5), 37.8 (C-2) ppm.

(E)-4-p-Tolylbut-3-enoic acid (1c)

(220 mg, 55\%)<smiles>Cc1ccc(/C=C/CC(=O)O)cc1</smiles> 
IR (film): $v_{\max }=3425,2958,2927,2871,1709,1514,1413,1180,967 \mathrm{~cm}^{-1}$.

${ }^{1} \mathrm{H}$ NMR $\left(400 \mathrm{MHz}, \mathrm{CDCl}_{3}\right): \delta=7.31(2 \mathrm{H}, \mathrm{d}, J 7.6 \mathrm{~Hz}, \mathrm{H}-6), 7.05(2 \mathrm{H}, \mathrm{d}, J 7.8 \mathrm{~Hz}, \mathrm{H}-7)$, $6.41(1 \mathrm{H}, \mathrm{d}, J 15.2, \mathrm{H}-4), 6.14(1 \mathrm{H}, \mathrm{m}, \mathrm{H}-3), 3.24(2 \mathrm{H}, \mathrm{t}, J 7.3 \mathrm{~Hz}, \mathrm{H}-2), 2.26(3 \mathrm{H}, \mathrm{s}, \mathrm{H}-9)$ ppm.

${ }^{13} \mathrm{C}$ NMR (63 MHz, $\mathrm{CDCl}_{3}$ ): $\delta=178.2$ (C-1), 137.5 (C-8), 133.9 (C-5), 129.3 (C-4), 129.1 (2C, C-7), 126.3 (2C, C-6), 119.8 (C-3), 38.0 (C-2), 21.2 (C-9) ppm.

MS: $m / z(\%): 176$ (48), 131 (94), 115 (100), 91 (57).

HRMS found $m / z(E I)=176.0839 \mathrm{C}_{11} \mathrm{H}_{12} \mathrm{O}_{2}$ calcd. 176.0837.

\section{(E)-4-(4-Bromophenyl)but-3-enoic acid (1d) ${ }^{1}$}

(298 mg, 55\%)

${ }^{1} \mathrm{H}$ NMR (400 MHz, $\left.\mathrm{CDCl}_{3}\right): \delta=7.70-7.75(2 \mathrm{H}, \mathrm{m}, \mathrm{H}-7), 7.35-7.40(2 \mathrm{H}, \mathrm{m}, \mathrm{H}-6), 6.62(1 \mathrm{H}$, d, J $15.9 \mathrm{~Hz}, \mathrm{H}-4), 6.37(1 \mathrm{H}, \mathrm{dd}, J 15.1,7.9 \mathrm{~Hz}, \mathrm{H}-3), 3.31(2 \mathrm{H}, \mathrm{d}, J 7.1 \mathrm{~Hz}, \mathrm{H}-2) \mathrm{ppm}$.

${ }^{13} \mathrm{C}$ NMR $\left(63 \mathrm{MHz}, \mathrm{CDCl}_{3}\right): \delta=177.9(\mathrm{C}-1), 135.6$ (C-5), 132.8 (C-4), 131.7 (2C, C-7), 127.7 (2C, C-6), 121.5 (C-3), 37.9 (C-2) ppm.

\section{(E)-4-(Naphthalen-3-yl)but-3-enoic acid (1e) ${ }^{2}$}

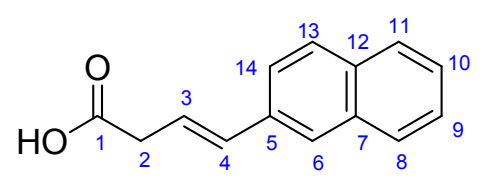

(297 mg, 63\%)

IR (film): $v_{\max }=3434,2359,1642 \mathrm{~cm}^{-1}$.

${ }^{1} \mathrm{H}$ NMR $\left(400 \mathrm{MHz}, \mathrm{CDCl}_{3}\right): \delta=7.36(3 \mathrm{H}, \mathrm{d}, \mathrm{J} 8.5 \mathrm{~Hz}, \mathrm{H}-9,10,14), 7.15-7.20(4 \mathrm{H}, \mathrm{m}, \mathrm{H}-$ 6,8,11,13), 6.39 (1H, d, J 15.9 Hz, H-4), 6.18-6.21 (1H, m, H-3), 3.23 (2H, d, J 7.0 Hz, H-2) ppm.

MS: $m / z$ (\%): 212 (82), 167 (100), 152 (61), 115 (15).

HRMS found $m / z\left(\right.$ EI-H $\left.^{+}\right)=212.0835 \mathrm{C}_{14} \mathrm{H}_{12} \mathrm{O}_{2}$ calcd. 212.0837 .

\section{(E)-5-(2-Methylnaphthalen-1-yl)pent-3-enoic acid (1f)}

(276 mg, 54\%)

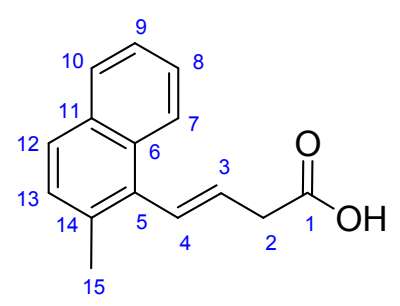

m.p. $=76-78{ }^{\circ} \mathrm{C}$

IR (film): $v_{\max }=2926,2851,2356,1705,1461,1373,1292,1223,1079 \mathrm{~cm}^{-1}$.

${ }^{1} \mathrm{H}$ NMR (400 MHz, CDCl $)$ ): $\delta=8.02(1 \mathrm{H}, \mathrm{d}, J 8.3 \mathrm{~Hz}, \mathrm{H}-7), 7.72(1 \mathrm{H}, \mathrm{d}, J 7.7 \mathrm{~Hz}, \mathrm{H}-10)$, 7.61 (1H, d, J 8.4 Hz, H-12), 7.32-7.42 (2H, m, H-8,9), 7.26 (1H, d, J 8.4 Hz, H-13), 6.78 $(1 \mathrm{H}, \mathrm{d}, J 16.1 \mathrm{~Hz}, \mathrm{H}-4), 5.88(1 \mathrm{H}, \mathrm{td}, J 16.0,7.1 \mathrm{~Hz}, \mathrm{H}-3), 3.40(2 \mathrm{H}, \mathrm{d}, J 7.1 \mathrm{~Hz}, \mathrm{H}-2), 2.41$ $(3 \mathrm{H}, \mathrm{s}, \mathrm{H}-15) \mathrm{ppm}$. 
${ }^{13} \mathrm{C}$ NMR (63 MHz, $\left.\mathrm{CDCl}_{3}\right): \delta=178.7$ (C-1), $133.1(\mathrm{C}-4), 131.0(2 \mathrm{C}, \mathrm{C}-5,14), 130.3(\mathrm{C}-11)$, 128.8 (C-6), 128.2 (C-10), 128.1 (C-8), 127.3 (C-12), 127.1 (C-13), 126.1 (C-9), 125.1 (C-7), 124.8 (C-3), 38.4 (C-2), 20.8 (C-15) ppm.

MS: $m / z(\%): 226(60), 181(18), 166(100), 152(22), 115$ (11).

HRMS found $m / z(\mathrm{EI})=226.0997, \mathrm{C}_{15} \mathrm{H}_{14} \mathrm{O}_{2}$ calcd. 226.0994 .

\section{General Procedure for the synthesis of 4-alkylbut-3-enoic acids 1g-1j:}

The aldehyde (10 mmol) and malonic acid (10 mmol, $1.04 \mathrm{~g})$ was mixed thoroughly with chromatography grade silica (200 mesh, $1 \mathrm{~g}$ ) and the resulting powder in a microwave tube was subjected to microwave irradiation $\left(300 \mathrm{~W}, 50{ }^{\circ} \mathrm{C}, 10 \mathrm{~min}\right)$. The reaction mixture was allowed to cool to room temperature and extracted with dichloromethane $(30 \mathrm{~mL})$. The solvent was removed under reduced pressure the compound was purified by flash chromatography eluting with petroleum ether:ethyl acetate:acetic acid $(90: 8: 2){ }^{3}$

\section{(E)-Hept-3-enoic acid (1g) ${ }^{4}$}

(313 $\mathrm{mg}, 21 \%)$

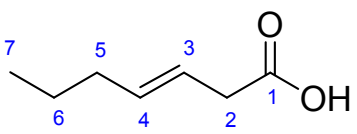

IR (film): $v_{\max }=2926,2876,1712,1411,1292,1224,1174,1123,967,936 \mathrm{~cm}^{-1}$.

${ }^{1} \mathrm{H} \mathrm{NMR}\left(400 \mathrm{MHz}, \mathrm{CDCl}_{3}\right): \delta=5.40-5.58(2 \mathrm{H}, \mathrm{m}, \mathrm{H}-3,4), 3.00(2 \mathrm{H}, \mathrm{d}, J 7.2 \mathrm{~Hz}, \mathrm{H}-2), 1.95$ $(2 \mathrm{H}, \mathrm{q}, J 6.8 \mathrm{~Hz}, \mathrm{H}-5), 1.27-1.39$ (2H, m, H-6), 0.82 (3H, t, $J 7.4 \mathrm{~Hz}, \mathrm{H}-7)$ ppm.

MS: $m / z$ (\%): 128 (13), $110(69), 68$ (100).

HRMS found $m / z(E I)=128.0833, \mathrm{C}_{7} \mathrm{H}_{12} \mathrm{O}_{2}$ calcd. 128.0837 .

\section{(E)-Oct-3-enoic acid (1h) $)^{4}$}

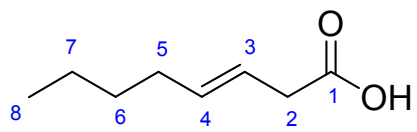

(391 mg, 28\%)

${ }^{1} \mathrm{H}$ NMR $\left(400 \mathrm{MHz}, \mathrm{CDCl}_{3}\right): \delta=5.40-5.58(2 \mathrm{H}, \mathrm{m}, \mathrm{H}-3,4), 3.02(2 \mathrm{H}, \mathrm{d}, J 6.7 \mathrm{~Hz}, \mathrm{H}-2), 1.95$ $(2 \mathrm{H}, \mathrm{q}, J 7.0 \mathrm{~Hz}, \mathrm{H}-5), 1.18-1.32(4 \mathrm{H}, \mathrm{m}, \mathrm{H}-6,7), 0.82(3 \mathrm{H}, \mathrm{t}, J 7.0 \mathrm{~Hz}, \mathrm{H}-8) \mathrm{ppm}$.

${ }^{13} \mathrm{C}$ NMR $\left(63 \mathrm{MHz}, \mathrm{CDCl}_{3}\right): \delta=179.0(\mathrm{C}-1), 135.5(\mathrm{C}-4), 120.7(\mathrm{C}-3), 37.9(\mathrm{C}-2), 32.1(\mathrm{C}-$ 5), $31.2(\mathrm{C}-6), 22.2(\mathrm{C}-7), 13.9(\mathrm{C}-8) \mathrm{ppm}$.

MS: $m / z(\%): 142(10), 124(86), 96(22), 82(100)$.

HRMS found $m / z(\mathrm{EI})=142.0995 \mathrm{C}_{9} \mathrm{H}_{16} \mathrm{O}_{2}$ calcd. 142.0994 .

\section{(E)-Non-3-enoic acid (1i) $)^{5}$}

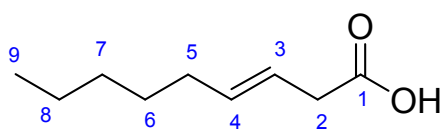

(908 mg, 66\%)

${ }^{1} \mathrm{H}$ NMR $\left(400 \mathrm{MHz}, \mathrm{CDCl}_{3}\right) \delta=5.40-5.58(2 \mathrm{H}, \mathrm{m}, \mathrm{H}-3,4), 3.02(2 \mathrm{H}, \mathrm{d}, J 6.7 \mathrm{~Hz}, \mathrm{H}-2), 1.95$ (2H, q, J $6.2 \mathrm{~Hz}, \mathrm{H}-5), 1.14-1.28$ (6H, m, H-6,7,8), 0.82 (3H, t, J $6.7 \mathrm{~Hz}, \mathrm{H}-9)$ ppm.

${ }^{13} \mathrm{C}$ NMR $\left(63 \mathrm{MHz}, \mathrm{CDCl}_{3}\right): \delta=178.7(\mathrm{C}-1), 135.6(\mathrm{C}-4), 120.6(\mathrm{C}-3), 37.8(\mathrm{C}-2), 32.4(\mathrm{C}-$ 5), 31.4 (C-7), 28.8 (C-6), 22.5 (C-8), 14.0 (C-9) ppm.

MS: $m / z(\%): 156(8), 138$ (65), 101 (23), 96 (49), 87 (51), 73 (81), 60 (100). 
HRMS found $m / z(\mathrm{EI})=156.1150, \mathrm{C}_{9} \mathrm{H}_{16} \mathrm{O}_{2}$ calcd. 156.1150 .

\section{(E)-Tetradec-3-enoic acid (1j)}

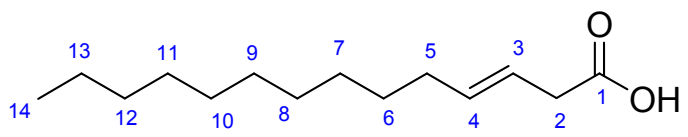

(373 mg, 35\%)

m.p. $=37-39^{\circ} \mathrm{C}$

IR (film): $v_{\max }=3432,1642 \mathrm{~cm}^{-1}$.

${ }^{1} \mathrm{H}$ NMR $\left(400 \mathrm{MHz}, \mathrm{CDCl}_{3}\right): \delta=5.49-5.69(2 \mathrm{H}, \mathrm{m}, \mathrm{H}-3,4), 3.09(2 \mathrm{H}, \mathrm{d}, J 6.6 \mathrm{~Hz}, \mathrm{H}-2)$, 2.03-2.10 (2H, m, H-5), 1.25-1.35 (16H, m, H-6,7,8,9,10,11,12,13), 0.92 (3H, t, H-14) ppm.

${ }^{13} \mathrm{C}$ NMR $\left(63 \mathrm{MHz}, \mathrm{CDCl}_{3}\right): \delta=178.9(\mathrm{C}-1), 135.5(\mathrm{C}-4), 120.2(\mathrm{C}-3), 37.9(\mathrm{C}-2), 34.5(\mathrm{C}-$ 5), 32.5 (C-6), 31.9 (C-7), 29.6 (C-8), 29.5 (C-9), 29.4 (C-10), 29.3 (C-11), 29.1 (C-12), 22.7 (C-13), $14.1(\mathrm{C}-14) \mathrm{ppm}$.

MS: $m / z$ (\%): 226 (20), 208 (94), 190 (41), 166 (70), 123 (56), 100 (62), 83 (68), 69 (100).

HRMS found $m / z\left(\mathrm{EI}^{+}\right)=226.1941, \mathrm{C}_{14} \mathrm{H}_{26} \mathrm{O}_{2}$ calcd. 226.1933 .

\section{General procedure for the catalytic reaction:}

To a solution of diphenyl diselenide ( $5 \mathrm{~mol} \%, 3.4 \mathrm{mg}, 0.01 \mathrm{mmol})$ in acetonitrile $(3 \mathrm{~mL})$ was added the $\beta, \gamma$-unsaturated acid $1(0.22 \mathrm{mmol})$, followed by bis(trifluoroacetoxy)iodobenzene (100 $\mathrm{mg}, 0.23 \mathrm{mmol}$ ) and the mixture stirred under argon at room temperature until TLC showed no remaining starting material. The solvent was then evaporated under reduced pressure and the residue was purified immediately by flash column chromatography eluting with ethyl acetate : light petroleum $(2: 8)$ to yield the cyclisation products.

\section{5-Phenylfuran-2(5H)-one (2a) ${ }^{6}$}

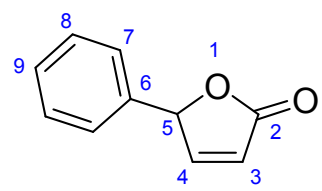

Colorless oil, $24 \mathrm{mg}, 70 \%$.

${ }^{1} \mathrm{H}$ NMR (400 MHz, $\left.\mathrm{CDCl}_{3}\right): \delta=7.53(1 \mathrm{H}, \mathrm{dd}, J 5.5,1.6 \mathrm{~Hz}, \mathrm{H}-4), 7.40-7.35$ (3H, m, H-7,9), 7.30-7.25 (2H, m, H-8), $6.23(1 \mathrm{H}, \mathrm{dd}, J 5.5,2.0 \mathrm{~Hz}, \mathrm{H}-5), 6.01(1 \mathrm{H}, \mathrm{dd}, J 2.0,1.6 \mathrm{~Hz}, \mathrm{H}-3)$ ppm.

${ }^{13} \mathrm{C}$ NMR (63 MHz, $\mathrm{CDCl}_{3}$ ): $\delta=173.1$ (C-2), 155.9 (C-4), 134.3 (C-6), 129.4 (C-9), 129.2 (2C, C-8), 126.5 (2C, C-7), 120.1 (C-3), 84.4 (C-5) ppm.

\section{5-Benzylfuran-2(5H)-one (2b) ${ }^{7}$}<smiles>COC1C=CC(=O)O1</smiles>

Colorless oil, $20 \mathrm{mg}, 59 \%$. 
${ }^{1} \mathrm{H}$ NMR $\left(400 \mathrm{MHz}, \mathrm{CDCl}_{3}\right): \delta=7.30(1 \mathrm{H}, \mathrm{dd}, J 5.7,1.5 \mathrm{~Hz}, \mathrm{H}-4), 7.25(2 \mathrm{H}, \mathrm{tt}, J 8.0,1.6 \mathrm{~Hz}$, H-9), 7.19 (2H, tt, J 5.9, $1.3 \mathrm{~Hz}, \mathrm{H}-8), 7.14(1 \mathrm{H}, \mathrm{d}, J 8.3 \mathrm{~Hz}, \mathrm{H}-10), 6.00$ (1 H, dd, J 5.7, 2.0, H-3), 5.15 (1H, m, H-5), 3.07 (1H, dd, $J 13.8,6.4 \mathrm{~Hz}, \mathrm{H}-6 \mathrm{a}), 2.89$ (1H, dd, $J 13.9,7.0$ Hz, H6b) ppm.

${ }^{13} \mathrm{C}$ NMR (63 MHz, $\left.\mathrm{CDCl}_{3}\right): \delta=172.8(\mathrm{C}-2), 155.6(\mathrm{C}-4), 134.8(\mathrm{C}-7), 129.4$ (2C, C-9), 128.7 (2C, C-8), 127.3 (C-10), 122.1 (C-3), 83.4 (C-5), 36.6 (C-6) ppm.

\section{5-p-Tolylfuran-2-(5H)-one $(2 c)^{8}$}

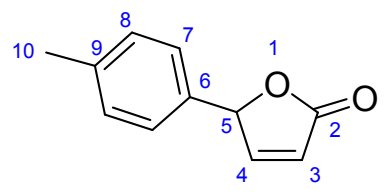

Colorless oil, $16 \mathrm{mg}, 57 \%$.

${ }^{1} \mathrm{H}$ NMR $\left(500 \mathrm{MHz}, \mathrm{CDCl}_{3}\right) \delta 7.47(1 \mathrm{H}, \mathrm{dd}, J$ 5.6, $1.6 \mathrm{~Hz}, \mathrm{H}-4), 7.13(2 \mathrm{H}, \mathrm{d}, J 8.1 \mathrm{~Hz}, \mathrm{H}-8)$, $7.01(2 \mathrm{H}, \mathrm{d}, J 7.9 \mathrm{~Hz}, \mathrm{H}-7), 6.16$ (1H, ddd, $J 6.68,5.65,2.11 \mathrm{~Hz}, \mathrm{H}-5), 5.92(1 \mathrm{H}, \mathrm{td}, J 17.9$, $1.81 \mathrm{~Hz}, \mathrm{H}-3), 2.29(3 \mathrm{H}, \mathrm{s}, \mathrm{H}-10) \mathrm{ppm}$.

${ }^{13} \mathrm{C}$ NMR (63 MHz, CDCl ${ }_{3}$ ) $\delta 172.0$ (C-2), 154.8 (C-4), 138.4 (C-6), 133.3 (C-9), 128.7 (2C, C-8) 128.1 (2C, C-7), 125.5 (C-3), 83.4 (C-5), 20.2 (C-10) ppm.

\section{5-(4-Bromophenyl)furan-2(5H)-one $(2 d)^{8}$}

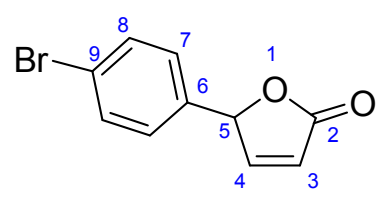

Colorless oil, $19 \mathrm{mg}, 54 \%$.

${ }^{1} \mathrm{H}$ NMR (400 MHz, $\left.\mathrm{CDCl}_{3}\right): \delta=7.80(1 \mathrm{H}, \mathrm{d}, J 8.1 \mathrm{~Hz}, \mathrm{H}-4), 7.51(2 \mathrm{H}, \mathrm{d}, J 5.5 \mathrm{~Hz}, \mathrm{H}-8)$, $7.10(2 \mathrm{H}, \mathrm{t}, J 7.8 \mathrm{~Hz}, \mathrm{H}-7), 6.22(1 \mathrm{H}, \mathrm{dd}, J 5.6,2.1 \mathrm{~Hz}, \mathrm{H}-5), 5.97(1 \mathrm{H}, \mathrm{d}, J 1.2 \mathrm{~Hz}, \mathrm{H}-3) \mathrm{ppm}$. ${ }^{13} \mathrm{C} \mathrm{NMR}\left(63 \mathrm{MHz}, \mathrm{CDCl}_{3}\right): \delta=173.1$ (C-2), 155.8 (C-4), 131.6 (2C, C-8), 129.1 (2C, C-7), 126.2 (C-3), 121.1 (C-9), 84.6 (C-5) ppm.

MS: $m / z$ (\%): 240 (50), 185 (100), 159 (50), 131 (35).

HRMS found $m / z(E I)=237.9625, \mathrm{C}_{10} \mathrm{H}_{7} \mathrm{O}_{2}{ }^{79} \mathrm{Br}$ calcd. 236.9629 .

\section{5-(Naphthalen-2-yl)furan-2(5H)-one (2e) ${ }^{7}$}

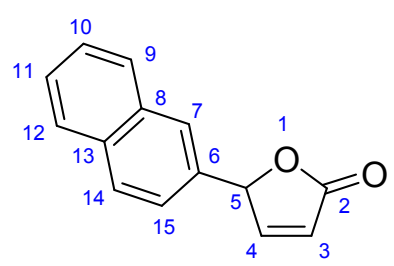

Colorless oil, $25 \mathrm{mg}, 60 \%$.

IR (film): $v_{\max }=1754,1158,1087,1026,901,818,749 \mathrm{~cm}^{-1}$.

${ }^{1} \mathrm{H}$ NMR (400 MHz, $\left.\mathrm{CDCl}_{3}\right) \delta$ 7.76-7.81 (3H, m, H-4,9,12), 7.70 (1H, s, H-7), 7.54 (1H, dd, $J$ 5.6, 1.7 Hz, H-14), 7.44-7.50 (2H, m, H-10,11), 7.23 (1H, dd, J 8.5, $1.7 \mathrm{~Hz}, \mathrm{H}-15), 6.25(1 \mathrm{H}$, dd, J 5.7, 2.1 Hz, H-5), $6.12(1 \mathrm{H}, \mathrm{s}, \mathrm{H}-3) \mathrm{ppm}$. 
${ }^{13} \mathrm{C}$ NMR $\left(63 \mathrm{MHz}, \mathrm{CDCl}_{3}\right): \delta=173.1(\mathrm{C}-2), 155.9(\mathrm{C}-4), 133.6(\mathrm{C}-6), 133.2(\mathrm{C}-8), 131.6$ (C-13), 129.1 (C-15), 128.19 (C-12), 127.8 (C-9), 126.9 (C-14), 126.8 (C-7), 126.2 (C-10), 123.3 (C-3), 121.1 (C-11), 84.5 (C-5) ppm.

MS: $m / z(\%): 210(88), 155$ (100), 127 (49).

HRMS found $m / z\left(\mathrm{EI}^{+}\right)=210.0679, \mathrm{C}_{14} \mathrm{H}_{10} \mathrm{O}_{2}$ calcd. 210.0681 .

\section{5-(2-Methylnaphthalen-y-1-yl)furan-2(5H)-one (2f)}

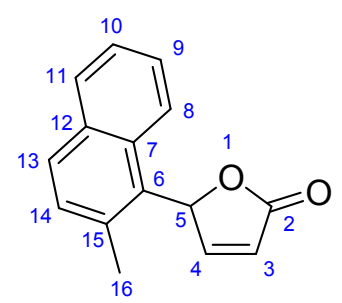

Colorless oil, $32 \mathrm{mg}, 65 \%$.

IR (film): $v_{\max }=1759,1157,1099,816 \mathrm{~cm}^{-1}$.

${ }^{1} \mathrm{H} \mathrm{NMR}\left(400 \mathrm{MHz} \mathrm{CDCl}_{3}\right): \delta=7.76(1 \mathrm{H}, \mathrm{t}, J 7.9 \mathrm{~Hz}, \mathrm{H}-4), 7.71(1 \mathrm{H}, \mathrm{d}, J 8.4 \mathrm{~Hz}, \mathrm{H}-8)$, 7.55-7.62 (1H, m, H-11) 7.34-7.43 (1H, m, H-13), 7.32-7.41 (1H, m, H-9), 7.12-7.23 (1H, m, H-10), 6.78-6.82 (1H, m, H-3), 6.30 (1H, dd, J 5.6, 2.5 Hz, H-5), 2.41 (3H, s, H-16) ppm. ${ }^{13} \mathrm{C}$ NMR $\left(63 \mathrm{MHz}, \mathrm{CDCl}_{3}\right): \delta=173.5(\mathrm{C}-2), 156.4(\mathrm{C}-4), 136.2(\mathrm{C}-6), 132.9(\mathrm{C}-15), 131.9$ (C-12), 130.2 (C-7), 130.0 (C-11), 129.0 (C-13), 127.0 (C-9), 125.2 (C-14), 124.8 (C-3), 122.4 (C-10), 121.9 (C-8), 82.0 (C-5), 20.8 (C-16) ppm.

MS: $m / z$ (\%): 224 (100), 195 (75), 165 (68), 141 (25), 115 (18).

HRMS found $m / z\left(\mathrm{EI}^{+}\right)=224.0837, \mathrm{C}_{15} \mathrm{H}_{112} \mathrm{O}_{2}$ calcd. 224.0837 .

\section{5-Propylfuran-2(5H)-one $(2 \mathrm{~g})^{9}$}

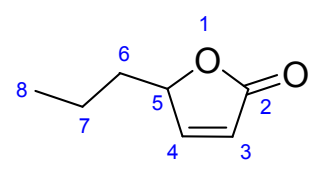

Colorless oil, $32 \mathrm{mg}, 65 \%$.

IR (film): $v_{\max }=2963,1748,1165,1114,818 \mathrm{~cm}^{-1}$.

${ }^{1} \mathrm{H}$ NMR $\left(400 \mathrm{MHz}, \mathrm{CDCl}_{3}\right): \delta=7.49(1 \mathrm{H}, \mathrm{dd}, J 5.7,1.4 \mathrm{~Hz}, \mathrm{H}-4), 6.03(1 \mathrm{H}, \mathrm{dd}, J 5.7,1.9$ Hz, H-5), 4.96-5.02 (1H, m, H-3), 1.69 (1H, m, H-6a), 1.59 (1H, m, H-6b), 1.40 (2H, m, H7), $0.90(3 \mathrm{H}, \mathrm{t}, J 7.4 \mathrm{~Hz}, \mathrm{H}-8) \mathrm{ppm}$.

${ }^{13} \mathrm{C} \mathrm{NMR}\left(63 \mathrm{MHz}, \mathrm{CDCl}_{3}\right): \delta=173.2(\mathrm{C}-2), 156.4(\mathrm{C}-4), 121.5$ (C-3), $83.2(\mathrm{C}-5), 35.2(\mathrm{C}-$ 6), $18.4(\mathrm{C}-7), 13.76(\mathrm{C}-8) \mathrm{ppm}$.

MS: $m / z(\%): 126.1(11), 97.1(100), 84.0(78)$

HRMS found $m / z\left(\mathrm{EI}^{+}\right)=126.0681, \mathrm{C}_{7} \mathrm{H}_{10} \mathrm{O}_{2}$ calcd. 126.0681 . 


\section{5-Butylfuran-2(5H)-one (2h)}

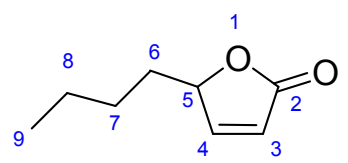

Colorless oil, $32 \mathrm{mg}, 65 \%$.

IR (film): $v_{\max }=2925,1753,1465,1164,820 \mathrm{~cm}^{-1}$.

${ }^{1} \mathrm{H} \mathrm{NMR}\left(400 \mathrm{MHz}, \mathrm{CDCl}_{3}\right): \delta=7.40(1 \mathrm{H}, \mathrm{dd}, J 5.8,1.5 \mathrm{~Hz}, \mathrm{H}-4), 6.05(1 \mathrm{H}, \mathrm{dd}, J 5.8,2.0$

Hz, H-5) 4.98-5.02 (1H, m, H-3) 1.65-1.75 (1H, m, H-6a), 1.55-1.65 (1H, m, H-6b), 1.23$1.40(4 \mathrm{H}, \mathrm{m}, \mathrm{H}-7,8), 0.85(3 \mathrm{H}, \mathrm{t}, J 7.1, \mathrm{H}-9) \mathrm{ppm}$.

${ }^{13} \mathrm{C}$ NMR $\left(63 \mathrm{MHz}, \mathrm{CDCl}_{3}\right): \delta=173.2(\mathrm{C}-2), 156.4(\mathrm{C}-4), 121.5(\mathrm{C}-3), 83.5(\mathrm{C}-5), 32.9(\mathrm{C}-$ 6), 27.0 (C-7), 22.4 (C-8), 13.8 (C-9) ppm.

MS: $m / z(\%): 140.1(9), 111.1(88), 84.0(100)$.

HRMS found $m / z\left(\mathrm{EI}^{+}\right)=140.0840, \mathrm{C}_{8} \mathrm{H}_{12} \mathrm{O}_{2}$ calcd. 140.0837 .

\section{5-Pentylfuran-2(5H)-one (2i)}

Colorless oil, $22 \mathrm{mg}, 49 \%$.

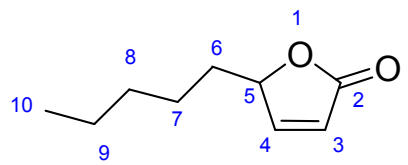

IR (film): $v_{\max }=2931,1753,1163,817 \mathrm{~cm}^{-1}$.

${ }^{1} \mathrm{H}$ NMR $\left(400 \mathrm{MHz}, \mathrm{CDCl}_{3}\right): \delta=7.40(1 \mathrm{H}, \mathrm{dd}, J 5.7,1.4 \mathrm{~Hz}, \mathrm{H}-4), 6.02(1 \mathrm{H}, \mathrm{dd}, J 5.7,2.0$

Hz, H-5) 4.94-5.02 (1H, m, H-3), 1.65-1.75 (1H, m, H-6a), 1.55-1.65 (1H, m, H-6b), 1.32 1.42 (2H, m, H-9) 1.20-1.30 (4H, m, H-7,8), 0.85 (3H, t, J 7.1 Hz, H-10) ppm.

${ }^{13} \mathrm{C}$ NMR $\left(63 \mathrm{MHz}, \mathrm{CDCl}_{3}\right): \delta=173.2(\mathrm{C}-2), 156.3(\mathrm{C}-4), 121.5(\mathrm{C}-3), 83.4(\mathrm{C}-5), 33.2(\mathrm{C}-$ 6), 31.5 (C-7), 24.6 (C-8), 22.4 (C-9), 13.9 (C-10) ppm.

MS: $m / z(\%): 154$ (4), 125 (70), 84 (100), 83 (20), 55 (15).

HRMS found $m / z\left(\mathrm{EI}^{+}\right)=154.0988, \mathrm{C}_{9} \mathrm{H}_{14} \mathrm{O}_{2}$ calcd. 154.0994.

\section{5-Decylfuran-2(5H)-one $(2 \mathrm{j})$}

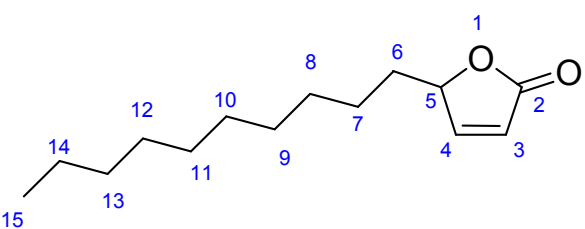

White solid, $48 \mathrm{mg}, 96 \%$.

$\mathrm{mp}=120-122^{\circ} \mathrm{C}$

IR (film): $v_{\max }=2920,2851,2360,1693,1467,965 \mathrm{~cm}^{-1}$.

${ }^{1} \mathrm{H} \mathrm{NMR}\left(400 \mathrm{MHz}, \mathrm{CDCl}_{3}\right): \delta=7.41(1 \mathrm{H}, \mathrm{dd}, J 5.7,1.4 \mathrm{~Hz}, \mathrm{H}-4), 6.05(1 \mathrm{H}, \mathrm{dd}, J 5.7,2.0$

$\mathrm{Hz}, \mathrm{H}-5), 4.98-5.02$ (1H, m, H-3), 1.65-1.75 (1H, m, H-6a), 1.55-1.65 (1H, m, H-6b), 1.351.45 (2H, m, H-14), 1.18-1.22 (14H, m, H-7,8,9,10,11,12,13) 0.82 (3H, t, J 6.7 Hz, H-15) ppm.

${ }^{13} \mathrm{C}$ NMR (63 MHz, $\left.\mathrm{CDCl}_{3}\right): \delta=174.4(\mathrm{C}-2), 157.0(\mathrm{C}-4), 121.4(\mathrm{C}-3), 84.2(\mathrm{C}-5), 33.0$ (C6), 32.6 (C-13), 29.6 (C-12), 29.5 (C-11), 29.4 (C-10), 29.3 (C-9), 29.1 (C-8), 24.6 (C-7), 22.1 (C-14), 14.1 (C-15) ppm.

MS: $m / z$ (\%): 224 (18), 164 (38), 97 (100), 84 (39), 69 (31).

HRMS found $m / z\left(\mathrm{EI}^{+}\right)=224.1778, \mathrm{C}_{14} \mathrm{H}_{24} \mathrm{O}_{2}$ calcd. 224.1776. 


\section{$N$-(5-Oxo-3-phenyltetrahydrofuran-2-yl)ethanamide (7)}

Colorless oil, $29 \mathrm{mg}, 28 \%$.<smiles>CC(=O)NC1OC(=O)CC1c1ccccc1</smiles>

IR (film): $v_{\max }=3299,1782,1678,1541,1372,1260,1166,943,826,732,698 \mathrm{~cm}^{-1}$.

${ }^{1} \mathrm{H} \mathrm{NMR}\left(400 \mathrm{MHz}, \mathrm{CDCl}_{3}\right): \delta=7.12-7.38(5 \mathrm{H}, \mathrm{m}, \mathrm{H}-1,2,3), 6.15(1 \mathrm{H}, \mathrm{t}, J 8.8 \mathrm{~Hz}, \mathrm{H}-8)$, $3.55(1 \mathrm{H}, \mathrm{q}, J 9.1 \mathrm{~Hz}, \mathrm{H}-5), 3.00(1 \mathrm{H}, \mathrm{dd}, J 8.8,17.6 \mathrm{~Hz}, \mathrm{H}-6 \mathrm{a}), 2.70(1 \mathrm{H}, \mathrm{dd}, J 10.7,17.6 \mathrm{~Hz}$, H-6b), 1.95 (3H, s, H-10) ppm.

${ }^{13} \mathrm{C}$ NMR (63 MHz, $\left.\mathrm{CDCl}_{3}\right): \delta=174.3(\mathrm{C}-7), 171.4(\mathrm{C}-9), 136.9(\mathrm{C}-4), 129.3(2 \mathrm{C}, \mathrm{C}-2)$, 128.2 (C-1), 127.1 (2C, C-3), 86.1 (C-8), 46.9 (C-5), 37.2 (C-6), 23.2 (C-10) ppm.

MS: $m / z(\%): 160$ (100), $178(58), 201$ (70), 220 (18).

HRMS found $m / z\left(\mathrm{M}+\mathrm{H}^{+}\right)=220.0979, \mathrm{C}_{12} \mathrm{H}_{14} \mathrm{NO}_{3}{ }^{+}$calcd. 220.0974 .

(1) Thibonnet, J.; Abarbri, M.; Parrain, J. L.; Duchêne, A. Tetrahedron 2003, 59, 4433-4441.

(2) Zhang, X.; Yao, X. T.; Dalton, T. J.; Shams, G.; Lei, L.; Patil, N. P.; Feller, R. D.; Hsu, F. L.; Cliff, G.; Miller, D. D. J. Med. Chem. 1996, 39, 3001-3013.

(3) Kumar, S. H. M.; Reddy, S. B. V.; Reddy, J. E.; Yadav, J. S. Tetrahedron Lett. 1999, 40, 2401-2404.

(4) Ragoussis, N.; Ragoussis, V. J. Chem. Soc., Perkin Trans. 1 1998, 3529-3533.

(5) Kawashima, M.; Sato, T.; Fujisawa, T. Bull. Chem. Soc. Jpn. 1988, 61, 3255-3264.

(6) Renard, M.; Ghosez, L. A. Tetrahedron 2001, 57, 2597-2608.

(7) Kang, J.; Lee, E.; Park, S.; Shin, S. Tetrahedron Lett. 2005, 46, 7431-7433.

(8) Bartlett, P. A. J. Am. Chem. Soc. 1959, 37, 2007-2022.

(9) Jefford, C. W.; Sledeski, A. W.; Boukouvales, J. J. Chem. Soc., Chem. Commun. 1988, 364-365. 